\title{
Unraveling the roles of vitamin $D$ status and melanin during COVID-19 (Review)
}

\author{
POLYTIMI SIDIROPOULOU ${ }^{1}$, ANCA OANA DOCEA ${ }^{2}$, VASILIKI NIKOLAOU ${ }^{1}$, \\ MARTHA-SPYRIDOULA KATSAROU ${ }^{3}$, DEMETRIOS A. SPANDIDOS ${ }^{4}$, ARISTIDIS TSATSAKIS ${ }^{5,6}$, \\ DANIELA CALINA $^{7}$ and NIKOLAOS DRAKOULIS ${ }^{3}$
}

${ }^{1} 1$ st Department of Dermatology-Venereology, Faculty of Medicine, National and Kapodistrian University of Athens,

'A. Sygros' Hospital for Skin and Venereal Diseases, 16121 Athens, Greece; ${ }^{2}$ Department of Toxicology, University of Medicine and Pharmacy of Craiova, 200349 Craiova, Romania; ${ }^{3}$ Research Group of Clinical Pharmacology and Pharmacogenomics, Faculty of Pharmacy, School of Health Sciences, National and Kapodistrian University of Athens, 15771 Athens; ${ }^{4}$ Laboratory of Clinical Virology and ${ }^{5}$ Department of Forensic Sciences and Toxicology, School of Medicine,

University of Crete, 71003 Heraklion, Greece; ${ }^{6}$ Department of Analytical Toxicology, Pharmaceutical Chemistry and Pharmacognosy, Sechenov University, 119991 Moscow, Russia; ${ }^{7}$ Department of Clinical Pharmacy, University of Medicine and Pharmacy of Craiova, 200349 Craiova, Romania

Received October 23, 2020; Accepted November 25, 2020

DOI: $10.3892 /$ ijmm.2020.4802

\begin{abstract}
As the coronavirus disease 2019 (COVID-19) continues to spread worldwide, it has become evident that the morbidity and mortality rates clearly vary across nations. Although several factors may account for this disparity, striking differences within and between populations indicate that ethnicity might impact COVID-19 clinical outcomes, reflecting the 'color of disease'. Therefore, the role of key biological variables that could interplay with viral spreading and severity indices has attracted increasing attention, particularly among non-Caucasian populations. Although the links between
\end{abstract}

Correspondence to: Dr Nikolaos Drakoulis, Research Group of Clinical Pharmacology and Pharmacogenomics, Faculty of Pharmacy, School of Health Sciences, National and Kapodistrian University of Athens, Panepistimioupolis, Zografou, 15771 Athens, Greece

E-mail: drakoulis@pharm.uoa.gr

Abbreviations: ACE2, angiotensin-converting enzyme 2; Ang II, angiotensin II; ARDS, acute respiratory distress syndrome; AT1R, AT1 receptor; BAME, Black, Asian and Minority Ethnic; CoV, coronavirus; COVID-19, coronavirus disease 2019; 7-DHC, 7-dehydrocholesterol; $\quad 1,25(\mathrm{OH})_{2} \mathrm{D}_{3}, \quad$ 1,25-dihydroxyvitamin $\mathrm{D}_{3}$; 25(OH)D, 25-hydroxyvitamin D; IFN- $\gamma$, interferon- $\gamma$; MasR, Mas receptor; NF- $\kappa \mathrm{B}$, nuclear factor- $\kappa \mathrm{B}$; RAS, renin-angiotensin system; ROS, reactive oxygen species; S, spike; SARS-CoV-2, severe acute respiratory syndrome coronavirus 2 ; Th, T helper; TLR, Toll-like receptor; TNF- $\alpha$, tumor necrosis factor- $\alpha$; Tregs, regulatory T cells; UV, ultraviolet; UVR, ultraviolet radiation; VDR, vitamin D receptor

Key words: coronavirus disease 2019, viral infection, skin pigmentation; immune response, immunity, ethnicity vitamin D status and the incidence and severity of COVID-19 remain elusive, several lines of emerging evidence suggest that vitamin D signaling, targeting several immune-mediated pathways, may offer potential benefits at different stages of SARS-CoV-2 infection. Given that the vitamin D status is modulated by several intrinsic and extrinsic factors, including skin type (pigmentation), melanin polymers may also play a role in variable COVID-19 outcomes among diverse population settings. Moreover, apart from the well-known limiting effects of melanin on the endogenous production of vitamin $\mathrm{D}$, the potential crosstalk between the pigmentary and immune system may also require special attention concerning the current pandemic. The present review article aimed to shed light on a range of mostly overlooked host factors, such as vitamin D status and melanin pigments, that may influence the course and outcome of COVID-19.

\section{Contents}

1. Introduction

2. SARS-CoV-2 infection

3. COVID-19 in skin of color

4. Potential role of melanin in the era of COVID-19

5. Vitamin D in respiratory antiviral defense

6. Implications of vitamin D for COVID-19

7. Conclusions

\section{Introduction}

Since the beginning of the 21st century, the emergence of severe acute respiratory syndrome coronavirus 2 (SARS-CoV-2, previously known as 2019-nCoV) has marked the third large-scale epidemic of a highly pathogenic coronavirus $(\mathrm{CoV})$ in the human 
population, following SARS-CoV in 2002 and the ongoing Middle East respiratory syndrome coronavirus (MERS-CoV) in 2012 (1,2). Coronavirus disease 2019 (COVID-19), an acute respiratory disease, has rapidly spread beyond its Chinese epicenter in Wuhan since late 2019, creating a public health burden of international concern.

While the COVID-19 pandemic continues to advance globally, the reports on clinical outcomes and risk factors for morbidity and mortality are increasingly emerging as the virus reaches new geographic areas. Despite the difficulties in cross-country comparisons, COVID-19 cases and mortality rates clearly vary around the globe $(3,4)$. Several factors may account for this discrepancy, including differences in population composition, age distribution, genetic predisposition, general health, healthcare access and socioeconomic status $(5,6)$. Although striking differences in SARS-CoV-2 infection within and between populations indicate that ethnicity may affect disease outcomes (7), little is known of the mechanisms through which genetic disparities could interplay with viral spreading and severity indices. As several vaccine prospects against SARS-CoV-2 are currently awaiting validation (8-12), the need to decrease COVID-19-related fatalities among vulnerable populations remains urgent. It is thus important to elucidate host factors that may influence susceptibility and/or response to SARS-CoV-2.

In this regard, interest has currently focused on the potential impact of vitamin D status on COVID-19 outcomes (13-15). In fact, it is already evident that the prevalence of vitamin D deficiency in Europe, particularly in the northern mid-latitudes, seems to be closely aligned to increased COVID-19 morbidity and mortality $(16,17)$. Moreover, certain non-white ethnic groups that are at higher risk of severe vitamin D deficiency [Black, Asian, and Minority Ethnic (BAME)] appear to be disproportionately affected by COVID-19 $(18,19)$.

Indeed, recent research has indicated that vitamin D signaling, mediating several antiviral and immune-enhancing pathways, may exert beneficial effects at different stages of COVID-19. The regulatory role of vitamin $\mathrm{D}$ in immune cell function, particularly by maintaining a dynamic balance between pro- and anti-inflammatory cytokine signals through modulating effects on the renin-angiotensin system (RAS), seems to be of particular importance in the context of severe COVID-19. Thus, although vitamin D deficiency may increase the risk of upper respiratory viral infections, it is the impact of the vitamin D status on cytokine profiles that is potentially much more relevant to the pathogenesis of COVID-19, pointing to a higher inflammatory response ('cytokine storm') in vitamin D-deficient individuals if exposed to SARS-CoV-2 (13-15,17,20).

Given the supportive role of vitamin D in immune responses against respiratory viruses (21), these observations require particular attention. Since vitamin D mainly results from endogenous skin production following exposure to ultraviolet (UV) solar radiation (22), skin pigmentation may thus play a role in non-white ethnic variations of COVID-19, as melanin can reduce the capacity of the skin to effectively absorb sunlight and synthesize vitamin D3. The present review article aimed to explore the evidence related to vitamin D status and melanin pigmentation that may have clinical implications on the course and outcomes of COVID-19.

\section{SARS-CoV-2 infection}

SARS-CoV-2 is an enveloped, positive-sense, and singlestranded RNA $\beta$-coronavirus that spreads mainly through the respiratory tract by exploiting the angiotensin-converting enzyme 2 (ACE2) as an entry receptor to infect lung alveolar and intestinal epithelial cells $(1,23)$. The initial binding between SARS-CoV-2 Spike (S) glycoprotein and ACE2 receptor on the surface of target cells is thus a critical step for virus endocytosis, determining the virus-host range and cellular tropism, as well as the virus-cell membrane fusion. ACE2, found in abundance in the human respiratory tract, has therefore taken center stage in the COVID-19 outbreak, as it can regulate both the cross-species and human-to-human transmission of SARS-CoV-2 (1).

The clinical spectrum of COVID-19 disease has been well documented. Infection with SARS-CoV-2 seems to follow a strikingly divergent course, ranging from non-symptomatic to life-threatening: The majority (80-85\%) of patients affected remain asymptomatic or display mild flu-like symptoms, while the remaining $15 \%$ develop severe disease and $5 \%$ of cases progress to a critical condition, with deaths mainly occurring in older and chronically ill patients as a result of acute respiratory distress syndrome (ARDS), sepsis and multiorgan failure $(24,25)$.

Of note, recent observational data have demonstrated an unusual risk factor pattern. While arterial hypertension and diabetes mellitus are the most commonly reported risk factors for more severe outcomes, patients with underlying lung conditions, i.e., chronic obstructive pulmonary disease, seem to be relatively protected from severe forms of COVID-19 (26). In addition, unlike influenza, a mild clinical course in the pediatric population has already been described. Curiously, the latter two groups are generally considered more vulnerable to respiratory pathogens $(15,26)$.

Although the immune response is vital for the control and resolution of SARS-CoV-2 infection (1), virus-host interactions trigger a diverse set of immune mediators against the invading virus, which is followed by immune overreaction and induction of a cytokine storm (excessive cytokine release) in at-risk individuals, as a known pathogenic event of ARDS development $(1,16)$. In this context, the RAS cascade has emerged at the forefront of COVID-19 pathophysiological mechanisms, although angiotensin II (Ang II) has been more well known for its cardiovascular and renal functions. Considering the ACE2 targeting by SARS-CoV-2, the RAS imbalance has been proposed to play a pivotal role in the pathogenesis of COVID-19. This hypothesis is mainly based on the reduction of transmembrane ACE2 as a result of enzyme endocytosis along with the S-glycoprotein of the virus. The consequent overstimulation of the classical ACE/Ang II/AT1 receptor (AT1R) axis in line with the downregulation of the anti-inflammatory ACE2/Ang-(1-7)/Mas Receptor (MasR) arm can compromise the depressor/pressor balance of RAS, resulting in a hyperinflammatory state that may partially be responsible for the severe complications associated with COVID-19 (26).

\section{COVID-19 in skin of color}

As the pandemic continues to spread worldwide, it has become evident that individuals of different ethnic backgrounds, but 
sharing a BAME origin appear to be more severely affected by COVID-19 compared to Caucasians (19). Increasing numbers of reports have demonstrated a pattern of higher risk for infection and adverse clinical outcomes from COVID-19 in ethnic minority groups (7,27-30). Recent UK data have revealed that over one-third of COVID-19 confirmed cases admitted to intensive care are of a BAME background (7). In addition, based on the UK Biobank data (2006-2010), both all-cause and COVID-19-related mortality rates have been estimated to be higher among ethnic minorities compared to the Caucasian British population during the first pandemic wave (19). Similarly, increased incidence and severity among African-American and minority communities have also been documented in the United States (31).

While the reason behind these disparities is probably multifactorial, involving a lower socioeconomic or medical comorbidity status, the disproportionate effect of COVID-19 on certain ethnic groups requires special attention. Although ethnicity may interplay with SARS-CoV-2 morbidity and mortality through different cultural, behavioral and social traits (7), exploring the mechanisms underlying the association between genetic variability and COVID-19 outcomes is obviously warranted by the evidence $(5,19)$.

Currently available data suggest that vitamin D deficiency may represent a potential mediator for poor COVID-19 outcomes in people of color (18). Given that highly melanized skin has long been shown to attenuate the cutaneous biosynthesis of vitamin D (32), indeed, key biological variables, including melanin, that may impact these observations should be considered.

\section{Potential role of melanin in the era of COVID-19}

Melanin pigments are considered the main drivers of human pigmentary status (33). Two types of melanin, i.e., eumelanin (brown/black) and pheomelanin (red/yellow) polymer, are produced within specific organelles (melanosomes) in epidermal melanocytes and are then transported into the surrounding keratinocytes. Specialized melanocytic enzymes and proteins are involved in melanin biosynthesis, with tyrosinase being the key enzyme catalyzing the initial step of melanogenesis, i.e., the oxidation and polymerization of the amino acid tyrosine to form the intermediate dopaquinone (33-35). Skin color diversity across individuals is mainly the result of differences in melanin content; the amounts of melanin in epidermal cells vary depending on the cutaneous phototype, being higher in dark and lower in light skin phenotypes (34-36).

Although skin, eye, and hair coloration is largely determined by genetics $(33,35)$, multiple intrinsic pathways (i.e., endocrine, immune, inflammatory signals) and extrinsic factors (i.e., UV light intensity, environmental pollution) are also involved in modulating the pigmentation patterns within and between populations (33,35-38). Notably, the localization of Ang II and its receptors in the skin, particularly the expression of functional AT1R in melanocytes, has been suggested to play a role in this regard. Apart from the well-established cardiovascular effects, Ang II has also been indicated to play an additional role in human skin pigmentation via the regulation of the melanogenic pathway (39-41).
Liu et al, exploring the effects of angiotensin on melanogenesis, recently demonstrated an increased tyrosinase activity and melanin content in human cultured melanocytes following AT1R stimulation in response to treatment with Ang II (39). These findings provide evidence to suggest an association between Ang II, tyrosinase and AT1R activation, supporting a stimulatory melanogenic effect of Ang II, which may be involved in cutaneous pigmentation.

Apart from defining an important phenotypic trait, melanin appears to play a major role in the natural photoprotection of skin $(42,43)$. In evolution, skin pigmentation in the human lineage has developed via a process of natural selection primarily to protect the skin from the damaging effects of solar UV radiation (UVR) (44). Since UVR can exert cytotoxic, mutagenic and immunosuppressive effects, either by direct action on DNA or indirectly by generating reactive oxygen species (ROS) and oxidative stress, the epidermis has been armed with melanin to maintain and/or restore local homeostasis against the UVR-driven insults $(37,42,43,45)$. Melanosomes, by forming supranuclear caps, protect keratinocytes from solar UV-induced DNA damage, while eumelanin acts as a direct scavenger of ROS generated upon UV exposure, reducing oxidative cellular damage $(34,42,43)$.

However, along with the well-known radical scavenging and antioxidant properties, an increased skin melanin content has long been recognized to be inversely related to the vitamin $\mathrm{D}$ status (32), which may probably account for the observed ethnic differences in vitamin D deficiency. Vitamin D is primarily obtained from 7-dehydrocholesterol (7-DHC) in the skin through solar exposure, with the UVB spectrum (290-315 nm) mostly contributing to its endogenous photosynthesis $(22,46,47)$. In fair-skinned individuals, short periods (20-30 min) of midday sun exposure 2-3 times per week is sufficient to achieve and maintain an optimal 25-hydroxyvitamin D [25(OH)D] status. However, this exposure pattern cannot be applied to darker skin populations (skin types V-VI), as well as the elderly who require higher weekly UVR doses to meet vitamin D needs. The equivalent exposure time or frequency for these specific groups has been estimated to be 2- to 10-fold higher compared to white-skinned, young Caucasians (47-49).

In fact, melanin can act as an effective natural filter by absorbing and scattering UVR, thereby impairing the solar UVB-mediated conversion of 7-DHC to previtamin $\mathrm{D}_{3}(32,45,47,50,51)$. As a result, the skin photosynthesis of $25(\mathrm{OH}) \mathrm{D}_{3}$ can be reduced by as much as $99 \%$ (32). This places dark-skinned individuals at higher risk for hypovitaminosis D than light-skinned ones and is particularly important in northern regions where pigmented skin (non-white ethnicity) is considered the major risk factor for vitamin D insufficiency/deficiency across all age groups $(47,50)$. Previous studies have consistently provided evidence to support the ethnic aspects of vitamin D inadequacy, demonstrating a higher prevalence in people with naturally darker skin (52-54). Of note, skin hyperpigmentation has also been recognized as a key risk factor for hypovitaminosis D in sunny lower latitudes, such as Australia (47).

Intriguingly, various biological functions affecting human health and disease have only recently been attributed to melanin pigments, but remain largely unexplored. In this respect, less clear is the link between melanin and immunity (55). However, 
accumulating evidence from several systems suggests that melanins are potent immunomodulators with both pro- and anti-inflammatory properties, depending on the type of melanin and host response (56). In humans, host melanin has long been implicated in the setting of ocular and gingival inflammatory disorders (57-59). Using a murine model, Kaya et al demonstrated an enhanced intraocular inflammatory response to uveitis in heavily pigmented eyes, possibly as a result of the pro-inflammatory effects of melanin (59). Similarly, in the human gingiva, a significant positive correlation between melanin distribution and presence of gingival inflammation has also been reported (57).

It should also be emphasized that melanin is considered to affect inflammatory responses directly and/or indirectly by influencing the host cytokine/chemokine production (56). Both in vitro and ex vivo data have indicated that melanin can modulate cytokine-mediated signaling cascades, increasing the release of pro-inflammatory mediators, such as interleukin (IL)-1, IL-6, interferon $\gamma$ (IFN- $\gamma$ ), and tumor necrosis factor- $\alpha(\mathrm{TNF}-\alpha)$. Additional evidence supporting a potential role of melanin in the course of host immune responses during infection is provided by in vitro findings demonstrating a melanin-induced activation of the nuclear factor- $\kappa \mathrm{B}(\mathrm{NF}-\kappa \mathrm{B})$ in monocytes through a Toll-like receptor (TLR)-dependent process $(55,60)$. A key question that remains to be addressed is whether or not these pathways could elicit an excessive immune response that may ultimately lead to tissue damage through a vigorous inflammatory reaction $(55,61)$.

Although the interactions between the pigmentary and immune system have not yet been fully elucidated, melanocytes, as a melanin source, have been reported to normally exist in oral and nasopharyngeal mucosa (62-64). These findings could thus pave the way for further consideration in different populations to translate heterogeneous basic research into a clinical perspective relevant to infectious disease, including SARS-CoV-2.

\section{Vitamin D in respiratory antiviral defense}

Several lines of evidence suggest that the vitamin D endocrine system is involved in multiple biologic processes and pathways, affecting not only musculoskeletal health, but also a variety of apparently different disease models, including infectious disease (46,65-67). Apart from its classical role in calcium and bone homeostasis, a modulatory role of vitamin $\mathrm{D}$ in immunity, inflammation and epithelial repair has previously been described $(68,69)$. The active metabolite 1,25-dihydroxyvitamin $\mathrm{D}_{3}\left[1,25(\mathrm{OH})_{2} \mathrm{D}_{3}\right]$ has long been recognized to possess immune regulatory properties. Vitamin D receptors (VDRs) are widely present in immunocompetent cells, such as antigen-presenting cells, T- and B-cells. By binding to VDR, $1,25(\mathrm{OH})_{2} \mathrm{D}_{3}$ modulates and downregulates adaptive, but enhances innate immunity and improves redox balance, thus counterbalancing inflammation on multiple levels $(70,71)$.

A growing number of studies have demonstrated that vitamin D can contribute to the defense against viral infections; notably, acute upper respiratory tract infections (21,72-80). Indeed, $1,25(\mathrm{OH})_{2} \mathrm{D}_{3}$ has been shown to exert antiviral effects, either directly by inhibiting viral replication, or via an anti-inflammatory and immunomodulatory mode of action $(69,81)$. Although the underlying mechanisms are very complex, vitamin D appears to support antiviral immunity by targeting three distinct pathways: physical barrier, cellular natural immunity, and adaptive immunity (79).

Vitamin D helps in preserving the epithelial intercellular junction integrity, which improves host mucosal defense against pathogen invasion $(15,68,82)$. At a cellular level, vitamin D metabolites have long been known to support innate antiviral responses in part by up-regulating antimicrobial peptides, such as human cathelicidin and defensins, to promote autophagy $(68-70,83)$. The adaptive immune effects of vitamin D include the inhibition of Th1/Th17 CD $4^{+}$T-cells and cytokines, such as TNF- $\alpha$ and IFN- $\gamma$, along with stimulatory actions on Th2 and regulatory T-cells (Tregs) $(69,70,84,85)$. By downregulating early pro-inflammatory signaling in favor of an anti-inflammatory Th2/Treg profile $(69,70,85), 1,25(\mathrm{OH})_{2} \mathrm{D}_{3}$ can suppress the altered cytokine milieu induced by viral and bacterial stimuli, as observed in COVID-19 patients, thus reducing the risk of extensive tissue damage due to uncontrolled inflammation (Fig. 1) (15).

Of note, vitamin D has also been shown to exert beneficial effects on local 'respiratory homeostasis' (69). Although several mechanisms may be involved in this regard, there are available data suggesting that vitamin D/VDR signaling may exert lung-protective effects at least partially by regulating the balance between key elements of the RAS (86-88). In fact, an inverse correlation between the vitamin D/VDR and RAS cascades has already been described. As vitamin D may act as a potent negative endocrine regulator of the RAS, vitamin D deficiency has been proposed as the other face of RAS overstimulation $(89,90)$. Considering that both systems have evolved in a similar and parallel manner, participating in the regulation of inflammatory and immunologic processes, as well as the presence of vitamin D (VDR) and RAS (AT1R) receptors in almost the same tissues, this link seems even more plausible (89).

Indeed, previous in vitro and in vivo experimental studies have demonstrated that the vitamin D/VDR pathway may trigger the ACE2/Ang-(1-7)/MasR axis, while inhibiting renin and the classical ACE/Ang II/AT1R cascade $(86,91,92)$. As ACE2 can directly exert lung-protective effects, whereas ACE exhibits an opposing function $(93,94)$, such evidence further supports the protective function of the vitamin D endocrine system in lung tissue (86-88). This feedback relation is also evident in other pathologies that are not discussed in this review but have been well documented, such as hypertension and chronic kidney disease (89).

\section{Implications of vitamin D for COVID-19}

Since vitamin D deficiency and/or insufficiency has emerged as a global pandemic linked to an increasing number of non-skeletal disorders, the importance of vitamin D/VDR signaling in overall health and well-being has attracted increasing attention in recent years $(65-67,95,96)$. Hypovitaminosis D has also been recognized as an independent risk factor for total mortality in the general population $(95,97)$.

Although randomized controlled trials and large-scale cohort studies investigating the links between the vitamin D status and COVID-19 incidence and severity are currently 


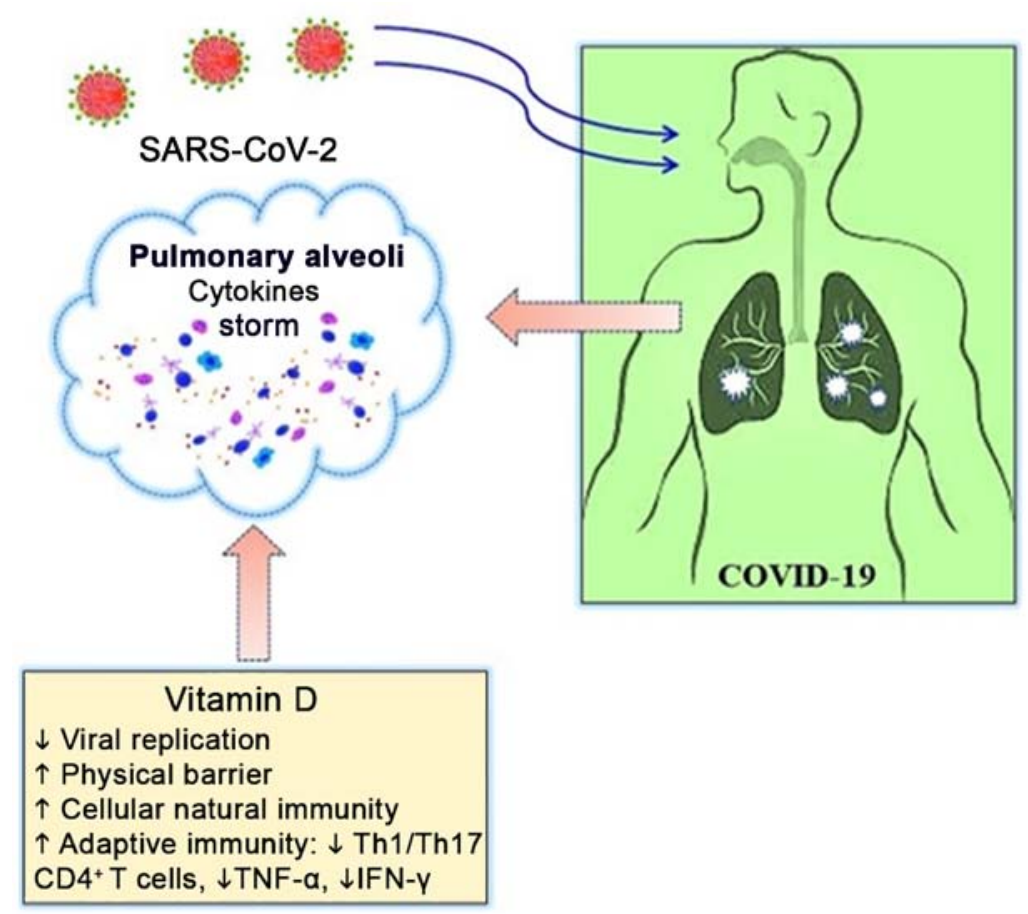

Figure 1. Potential antiviral mechanisms of vitamin D in COVID-19. COVID-19, coronavirus disease 2019; IFN- $\gamma$, interferon- $\gamma$; SARS-CoV-2, severe acute respiratory syndrome coronavirus 2 ; Th, T helper; TNF- $\alpha$, tumor necrosis factor- $\alpha$.

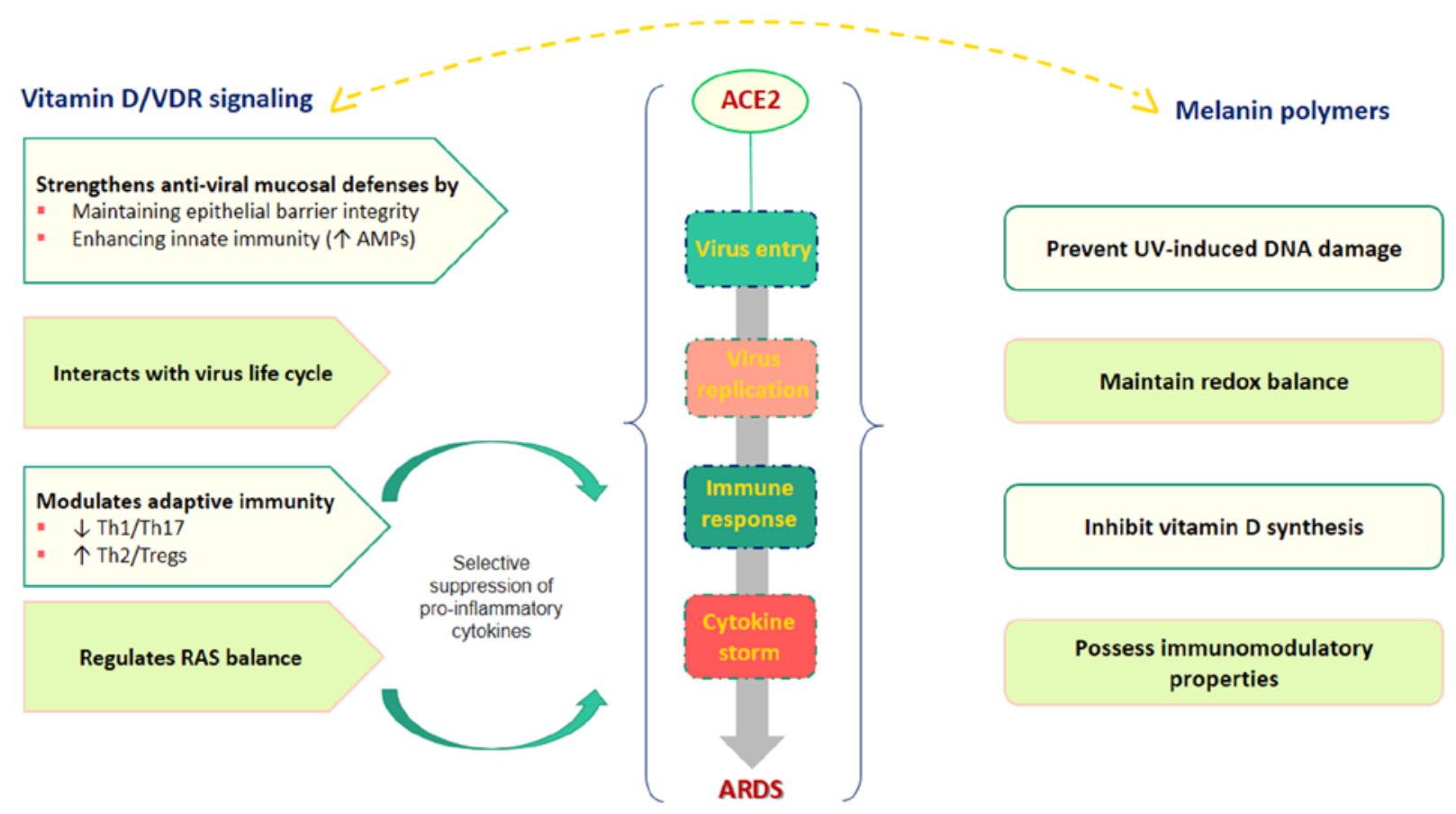

Figure 2. Schematic diagram summarizing the vitamin D/VDR and melanin related signaling pathways, as well as their potential implications for COVID-19. ACE2, angiotensin-converting enzyme 2; AMPs, antimicrobial peptides; ARDS, acute respiratory distress syndrome; RAS, renin-angiotensin system; Th, T helper; Tregs, regulatory T cells; UV, ultraviolet; VDR, vitamin D receptor.

limited, evolving epidemiological evidence supports the hypothesis that vitamin D inadequacy can negatively affect COVID-19 outcomes $(14,15,20,98)$.

The outbreak and peak of SARS-CoV-2 in wintertime, when vitamin D levels drop to their lowest, as well as the pattern of geographical spread of COVID-19 seem to reflect higher population rates of vitamin D deficiency $(15,20,98)$.
Of note, COVID-19 associated fatality rates appear to coincide with vitamin D deficiency rates, with northern mid-latitude countries, where vitamin D deficiency is still widely prevalent, bearing a greater burden of morbidity and mortality $(15,20,98)$.

Moreover, a striking overlap between the high-risk groups for severe COVID-19 and vitamin D insufficiency has already 
been reported. Indeed, severe COVID-19 infection and hypovitaminosis D appear to share numerous risk factors, including advanced age, male sex, obesity, darker skin pigmentation, inadequate sunlight exposure, and chronic disease comorbidity, particularly hypertension, cardiovascular disease, and diabetes $(14-16,98,99)$.

To further support this hypothesis, several clinical and observational studies have thus far demonstrated an inverse correlation between vitamin D status and COVID-19-associated morbidity and mortality $(15-17,20,100)$. Cross-sectional analyses in 20 European countries have reported a significant negative association between the mean vitamin $\mathrm{D}$ levels and the number of COVID-19 cases/1 million population (16) and between the average vitamin D levels and COVID-19-related deaths/1 million population (100). A recent review comprising 188 studies (47 original human research studies) on the relation between vitamin D and COVID-19 also provided biological plausibility supporting the assertions that vitamin D deficiency can explain every major risk factor, including the mystery of why elderly males and individuals with naturally melanin-rich skin are especially vulnerable, as well as every complication of COVID-19 (15).

From a biological perspective, there is compelling evidence to indicate that the vitamin D/VDR pathway can favorably modulate the host immunity to SARS-CoV-2, both in the early viraemic and later hyperinflammatory stages of COVID-19. In fact, vitamin D deficiency seems to compromise innate immune functions, increasing the risk of viral infections in the respiratory epithelium, including COVID-19 (14,15). Despite the sparse laboratory data regarding the impact of vitamin D on host responses to SARS-CoV-2, a recent in vitro study explored four compound libraries for antiviral activity demonstrating a direct inhibitory effect of calcitriol (the active form of vitamin D) on human nasal epithelial cells infected with SARS-CoV-2 (101).

However, it is the impact of vitamin D on unregulated cytokine production and, potentially, on the severity/risk of ARDS that is of particular importance in COVID-19 $(15,17,102)$. In this respect, the finding that vitamin $\mathrm{D}$ deficiency may increase the potential for cytokine storm by deregulating the $\mathrm{X}$-chromosome-linked RAS appears to be much more specific in the context of severe COVID-19, where overactivation of the RAS has been associated with a poorer prognosis $(14,15)$.

Although conclusive scientific data may eventually be available, such correlational evidence might be of great interest in darker-skinned individuals, who are more likely to be vitamin D deficient, as it points toward an aberrant inflammatory response if exposed to SARS-CoV-2, possibly indicating a higher risk of COVID-19 adverse outcomes.

A schematic diagram of the vitamin D/VDR and melanin related signaling pathways along with their potential implications for COVID-19 is presented in Fig. 2.

\section{Conclusions}

In summary, the present review attempts to broaden current knowledge of host biological factors, such as vitamin D status and melanin polymers, possibly related to clinical outcomes of COVID-19. Although contradictory data exist, vitamin D may turn into an effective adjuvant to mitigate the impact of the current pandemic, especially in populations where hypovitaminosis D is prevalent. Notably, the concept of vitamin D regulation of cytokine storm through the RAS opens up new perspectives on the functions of vitamin D/VDR signaling, providing a basis for exploring the potential use of vitamin $\mathrm{D}$ analogs in the prevention and/or treatment of COVID-19.

Given that vitamin D is a safe, inexpensive, and widely available agent, even in countries with limited resources, vitamin $\mathrm{D}$ inadequacy is obviously an easily modifiable risk factor. Therefore, from the literature reviewed here, prevention and/or restoration of vitamin D deficiency/insufficiency through vitamin D supplementation during the COVID-19 period seems to be highly supported by the evidence.

For greater benefits, however, consideration of basic biological variables, especially in different population settings, is clearly warranted as the COVID-19 infection rates are once again on the rise. At this point, our study might provide early insights into a range of mostly overlooked host factors throughout the disease pathway. Besides the well-known limiting effects of melanin on the cutaneous biosynthesis of vitamin $\mathrm{D}$, which could negatively affect COVID-19 outcomes, the potential interplay between the pigmentary and immune system may also require special emphasis regarding the current pandemic. Further research is needed to address these observations and elucidate whether any of the implicated effects could be specific to SARS-CoV-2.

\section{Acknowledgements}

Not applicable.

\section{Funding}

No funding was received.

\section{Availability of data and materials}

Not applicable.

\section{Authors' contributions}

DC conceived the presented idea, while PS retrieved the data and wrote the manuscript under the supervision of ND. ND aided in data extraction and revised the manuscript critically. PS and AOD designed the figures. AOD, VN, MSK, DAS and AT contributed to the editing and revision of the manuscript. All authors have read and agreed to the final version of the manuscript.

\section{Ethics approval and consent to participate}

Not applicable.

\section{Patient consent for publication}

Not applicable.

\section{Competing interests}

DAS is the Editor-in-Chief for the journal, but had no personal involvement in the reviewing process, or any influence in 
terms of adjudicating on the final decision, for this article. The other authors declare that they have no competing interests.

\section{References}

1. Guo YR, Cao QD, Hong ZS, Tan YY, Chen SD, Jin HJ, Tan KS, Wang DY and Yan Y: The origin, transmission and clinical therapies on coronavirus disease 2019 (COVID-19) outbreak - an update on the status. Mil Med Res 7: 11, 2020.

2. Docea AO, Tsatsakis A, Albulescu D, Cristea O, Zlatian O, Vinceti M, Moschos SA, Tsoukalas D, Goumenou M, Drakoulis N, et al: A new threat from an old enemy: Re-emergence of coronavirus (Review). Int J Mol Med 45: 1631-1643, 2020.

3. Lai CC, Wang CY, Wang YH, Hsueh SC, Ko WC and Hsueh PR: Global epidemiology of coronavirus disease 2019 (COVID-19): Disease incidence, daily cumulative index, mortality, and their association with country healthcare resources and economic status. Int J Antimicrob Agents 55: 105946, 2020.

4. Goumenou M, Sarigiannis D, Tsatsakis A, Anesti O Docea AO, Petrakis D, Tsoukalas D, Kostoff R, Rakitskii V, Spandidos DA, et al: COVID-19 in Northern Italy: An integrative overview of factors possibly influencing the sharp increase of the outbreak (Review). Mol Med Rep 22: 20-32, 2020.

5. Stopsack KH, Mucci LA, Antonarakis ES, Nelson PS and Kantoff PW: TMPRSS2 and COVID-19: Serendipity or Opportunity for Intervention? Cancer Discov 10: 779-782, 2020.

6. Tsatsakis A, Petrakis D, Nikolouzakis TK, Docea AO, Calina D, Vinceti M, Goumenou M, Kostoff RN, Mamoulakis C, Aschner M, et al: COVID-19, an opportunity to reevaluate the correlation between long-term effects of anthropogenic pollutants on viral epidemic/pandemic events and prevalence. Food Chem Toxicol 141: 111418, 2020.

7. Pareek M, Bangash MN, Pareek N, Pan D, Sze S, Minhas JS, Hanif W and Khunti K: Ethnicity and COVID-19: An urgent public health research priority. Lancet 395: 1421-1422, 2020.

8. Nitulescu GM, Paunescu H, Moschos SA, Petrakis D, Nitulescu G, Ion GND, Spandidos DA, Nikolouzakis TK, Drakoulis $\mathrm{N}$ and Tsatsakis A: Comprehensive analysis of drugs to treat SARS-CoV-2 infection: Mechanistic insights into current COVID-19 therapies (Review). Int J Mol Med 46: $467-488,2020$

9. Calina D, Sarkar C, Arsene AL, Salehi B, Docea AO, Mondal M, Islam MT, Zali A and Sharifi-Rad J: Recent advances, approaches and challenges in targeting pathways for potential COVID-19 vaccines development. Immunol Res 68: 315-324, 2020.

10. Kostoff RN, Kanduc D, Porter AL, Shoenfeld Y, Calina D, Briggs MB, Spandidos DA and Tsatsakis A: Vaccine- and natural infection-induced mechanisms that could modulate vaccine safety. Toxicol Rep 7: 1448-1458, 2020.

11. Calina D, Docea AO, Petrakis D, Egorov AM, Ishmukhametov AA, Gabibov AG, Shtilman MI, Kostoff R, Carvalho F, Vinceti M, et al: Towards effective COVID-19 vaccines: Updates, perspectives and challenges (Review). Int J Mol Med 46: 3-16,2020

12. Calina D, Hartung T, Docea AO, Spandidos DA, Egorov AM, Shtilman MI, Carvalho F and Tsatsakis A: COVID-19 vaccines: Ethical framework concerning human challenge studies. Daru: Aug 27, 2020 (Epub ahead of print).

13. Arboleda JF, Urcuqui-Inchima S and Vitamin D: Vitamin D Supplementation:APotential Approach for Coronavirus/COVID-19 Therapeutics? Front Immunol 11: 1523, 2020.

14. Martineau AR and Forouhi NG: Vitamin D for COVID-19: A case to answer? Lancet Diabetes Endocrinol 8: 735-736, 2020.

15. Benskin LL: A Basic Review of the Preliminary Evidence That COVID-19 Risk and Severity Is Increased in Vitamin D Deficiency. Front Public Health 8: 513, 2020.

16. Ali N: Role of vitamin D in preventing of COVID-19 infection, progression and severity. J Infect Public Health 13: 1373-1380, 2020.

17. Laird E, Rhodes J and Kenny RA: Vitamin D and inflammation: potential implications for severity of Covid-19. Ir Med J 113: 81, 2020.

18. Darling AL, Ahmadi KR, Ward KA, Harvey NC, Alves AC, Dunn-Waters DK, Lanham-New SA, Cooper C and Blackbourn DJ: Vitamin D status, body mass index, ethnicity and COVID-19: Initial analysis of the first-reported UK Biobank COVID-19 positive cases (n 580) compared with negative controls (n 723). MedRxiv: doi: https://doi.org/10.1101/2020.04 29.20084277 .
19. Patel P, Hiam L, Sowemimo A, Devakumar D and McKee M: Ethnicity and covid-19. BMJ 369: $\mathrm{m} 2282,2020$.

20. Grant WB, Lahore H, McDonnell SL, Baggerly CA, French CB, Aliano JL and Bhattoa HP: Evidence that Vitamin D Supplementation Could Reduce Risk of Influenza and COVID-19 Infections and Deaths. Nutrients 12: 988, 2020.

21. Martineau AR, Jolliffe DA, Hooper RL, Greenberg L, Aloia JF, Bergman P, Dubnov-Raz G, Esposito S, Ganmaa D, Ginde AA, et al: Vitamin D supplementation to prevent acute respiratory tract infections: systematic review and meta-analysis of individual participant data. BMJ 356: i6583, 2017.

22. Pike JW and Christakos S: Biology and mechanisms of action of the vitamin D hormone. Endocrinol Metab Clin North Am 46: 815-843, 2017.

23. Zhu N,Zhang D, Wang W, Li X, Yang B, Song J, Zhao X, Huang B, Shi W, Lu R, et al; China Novel Coronavirus Investigating and Research Team: A novel coronavirus from patients with pneumonia in China, 2019. N Engl J Med 382: 727-733, 2020

24. Huang C, Wang Y, Li X, Ren L, Zhao J, Hu Y, Zhang L, Fan G, Xu J, Gu X, et al: Clinical features of patients infected with 2019 novel coronavirus in Wuhan, China. Lancet 395: 497-506, 2020.

25. Tsatsakis A, Calina D, Falzone L, Petrakis D, Mitrut R, Siokas V, Pennisi M, Lanza G, Libra M, Doukas SG, et al: SARS-CoV-2 pathophysiology and its clinical implications: An integrative overview of the pharmacotherapeutic management of COVID-19. Food Chem Toxicol 146: 111769, 2020.

26. Lanza K, Perez LG, Costa LB, Cordeiro TM, Palmeira VA, Ribeiro VT, Simões E Silva AC and Silva AC: Covid-19: The renin-angiotensin system imbalance hypothesis. Clin Sci (Lond) 134: 1259-1264, 2020.

27. Aldridge RW, Lewer D, Katikireddi SV, Mathur R, Pathak N, Burns R, Fragaszy EB, Johnson AM, Devakumar D, Abubakar I, et al: Black, Asian and Minority Ethnic groups in England are at increased risk of death from COVID-19: Indirect standardisation of NHS mortality data. Wellcome Open Res 5: $88,2020$.

28. Khunti K, Singh AK, Pareek M and Hanif W: Is ethnicity linked to incidence or outcomes of covid-19? BMJ 369: m1548, 2020.

29. Pan D, Sze S, Minhas JS, Bangash MN, Pareek N, Divall P, Williams CM, Oggioni MR, Squire IB, Nellums LB, et al: The impact of ethnicity on clinical outcomes in COVID-19: A systematic review. EClinicalMedicine 23: 100404, 2020.

30. Sze S, Pan D, Gray LJ, Nevill CR, Martin CA, Nazareth J, Minhas JS, Divall P, Khunti K, Abrams K, et al: Ethnicity and clinical outcomes in COVID-19: A systematic review and meta-analysis. EClinicalMedicine: Nov 12, 2020 (Epub ahead of print).

31. Vahidy FS, Nicolas JC, Meeks JR, Khan O, Pan A, Jones SL, Masud F, Sostman HD, Phillips R, Andrieni JD, et al: Racial and ethnic disparities in SARS-CoV-2 pandemic: Analysis of a COVID-19 observational registry for a diverse US metropolitan population. BMJ Open 10: e039849, 2020.

32. Clemens TL, Adams JS, Henderson SL and Holick MF: Increased skin pigment reduces the capacity of skin to synthesise vitamin D3. Lancet 1: 74-76, 1982.

33. Pavan WJ and Sturm RA: The Genetics of Human Skin and Hair Pigmentation. Annu Rev Genomics Hum Genet 20: 41-72, 2019.

34. Abdel-Malek ZA and Swope VB: Epidermal melanocytes: regulation of their survival, proliferation, and function in human skin. In: Melanoma Development. Springer, Vienna, pp7-33, 2011.

35. Rees JL: Genetics of hair and skin color. Annu Rev Genet 37: 67-90, 2003.

36. Del Bino S, Duval C and Bernerd F: Clinical and biological characterization of skin pigmentation diversity and its consequences on UV impact. Int J Mol Sci 19: 2668, 2018.

37. Costin GE and Hearing VJ: Human skin pigmentation: Melanocytes modulate skin color in response to stress. FASEB J 21: 976-994, 2007.

38. Serre C, Busuttil V and Botto JM: Intrinsic and extrinsic regulation of human skin melanogenesis and pigmentation. Int $\mathrm{J}$ Cosmet Sci 40: 328-347, 2018.

39. Liu LH, Fan X, Li HT, An XX and Yang RY: Angiotensin II promotes melanogenesis via angiotensin II type 1 receptors in human melanocytes. Mol Med Rep 12: 651-656, 2015.

40. Liu LH, Fan X, Xia ZK, An XX and Yang RY: Angiotensin II stimulates melanogenesis via the protein kinase $C$ pathway. Exp Ther Med 10: 1528-1532, 2015.

41. Steckelings UM, Wollschläger T, Peters J, Henz BM, Hermes B and Artuc M: Human skin: Source of and target organ for angiotensin II. Exp Dermatol 13: 148-154, 2004. 
42. Brenner $\mathrm{M}$ and Hearing VJ: The protective role of melanin against UV damage in human skin. Photochem Photobiol 84: 539-549, 2008

43. Solano F: Photoprotection and skin pigmentation: melanin-related molecules and some other new agents obtained from natural sources. Molecules 25: 1537, 2020.

44. Rocha J: The evolutionary history of human skin pigmentation. J Mol Evol 88: 77-87, 2020.

45. Slominski A and Postlethwaite AE: Skin under the sun: when melanin pigment meets vitamin D. Endocrinology 156: 1-4, 2015.

46. Bikle DD: Vitamin D metabolism, mechanism of action, and clinical applications. Chem Biol 21: 319-329, 2014

47. Pearce SH and Cheetham TD: Diagnosis and management of vitamin D deficiency. BMJ 340: b5664, 2010.

48. Webb AR, Kazantzidis A, Kift RC, Farrar MD, Wilkinson J and Rhodes LE: Meeting vitamin D requirements in White Caucasians at UK latitudes: Providing a choice. Nutrients 10 497, 2018.

49. Farrar MD, Kift R, Felton SJ, Berry JL, Durkin MT, Allan D, Vail A, Webb AR and Rhodes LE: Recommended summer sunlight exposure amounts fail to produce sufficient vitamin D status in UK adults of South Asian origin. Am J Clin Nutr 94: $1219-1224,2011$

50. Bonilla C, Ness AR, Wills AK, Lawlor DA, Lewis SJ and Davey Smith G: Skin pigmentation, sun exposure and vitamin D levels in children of the Avon Longitudinal Study of Parents and Children. BMC Public Health 14: 597, 2014.

51. Hameed A and Akhtar N: The skin melanin: an inhibitor of vitamin-D3 biosynthesis: with special emphasis with structure of skin. A mini review. Dermatol Case Rep 4: 1, 2019.

52. Richard A, Rohrmann S and Quack Lötscher KC: Prevalence of vitamin D deficiency and its associations with skin color in pregnant women in the first trimester in a sample from Switzerland. Nutrients 9: 260, 2017.

53. Alzaman NS, Dawson-Hughes B, Nelson J, D'Alessio D and Pittas AG: Vitamin D status of black and white Americans and changes in vitamin D metabolites after varied doses of vitamin D supplementation. Am J Clin Nutr 104: 205-214, 2016.

54. Harris SS: Vitamin D and African Americans. J Nutr 136 1126-1129, 2006

55. ElObeid AS, Kamal-Eldin A, Abdelhalim MAK and Haseeb AM: Pharmacological properties of melanin and its function in health Basic Clin Pharmacol Toxicol 120: 515-522, 2017.

56. Mednick AJ, Nosanchuk JD and Casadevall A: Melanization of Cryptococcus neoformans affects lung inflammatory responses during cryptococcal infection. Infect Immun 73: 2012-2019, 2005.

57. Patsakas A, Demetriou N and Angelopoulos A: Melanin pigmentation and inflammation in human gingiva. J Periodontol 52 701-704, 1981.

58. Smith JR, Rosenbaum JT and Williams KA: Experimental melanin-induced uveitis: Experimental model of human acute anterior uveitis. Ophthalmic Res 40: 136-140, 2008.

59. Kaya M, Edward DP, Tessler H and Hendricks RL: Augmentation of intraocular inflammation by melanin. Invest Ophthalmol Vis Sci 33: 522-531, 1992

60. Pugh ND, Balachandran P, Lata H, Dayan FE, Joshi V, Bedir E, Makino T, Moraes R, Khan I and Pasco DS: Melanin: Dietary mucosal immune modulator from Echinacea and other botanical supplements. Int Immunopharmacol 5: 637-647, 2005.

61. Wilms H, Rosenstiel P, Sievers J, Deuschl G, Zecca L and Lucius R: Activation of microglia by human neuromelanin is NF-kappaB dependent and involves p38 mitogen-activated protein kinase: Implications for Parkinson's disease. FASEB J 17: 500-502, 2003.

62. Feller L, Masilana A, Khammissa RA, Altini M, Jadwat Y and Lemmer J: Melanin: the biophysiology of oral melanocytes and physiological oral pigmentation. Head Face Med 10: 8, 2014.

63. Fritz MA, Roehm PC, Bannan MA and Lalwani AK: Extracellular and intracellular melanin in inflammatory middle ear disease. Laryngoscope 124: E241-E244, 2014.

64. Na JY, Kim YH, Choi YD and Lee JS: Melanotic oncocytic metaplasia of the nasopharynx: A report of three cases and review of the literature. Korean J Pathol 46: 201-204, 2012.

65. Hossein-nezhad A and Holick MF: Vitamin D for health: A global perspective. Mayo Clin Proc 88: 720-755, 2013.

66. Wacker M and Holick MF: Sunlight and vitamin D: A global perspective for health. Dermatoendocrinol 5: 51-108, 2013.
67. Holick MF: Vitamin D: Extraskeletal health. Endocrinol Metab Clin North Am 39: 381-400, 2010

68. Schwalfenberg GK: A review of the critical role of vitamin D in the functioning of the immune system and the clinical implications of vitamin D deficiency. Mol Nutr Food Res 55: 96-108, 2011.

69. Zdrenghea MT, Makrinioti H, Bagacean C, Bush A, Johnston SL and Stanciu LA: Vitamin D modulation of innate immune responses to respiratory viral infections. Rev Med Virol 27: e1909, 2017.

70. Prietl B, Treiber G, Pieber TR and Amrein K: Vitamin D and immune function. Nutrients 5: 2502-2521, 2013.

71. Hoeck AD and Pall ML: Will vitamin D supplementation ameliorate diseases characterized by chronic inflammation and fatigue? Med Hypotheses 76: 208-213, 2011.

72. Jolliffe D, Camargo CA, Sluyter J, Aglipay M, Aloia J, Bergman P, Damsgaard C, Dubnov-Raz G, Esposito S, Ganmaa D, et al: Vitamin D supplementation to prevent acute respiratory infections: systematic review and meta-analysis of aggregate data from randomised controlled trials. medRxiv: doi: https:// doi.org/10.1101/2020.07.14.20152728.

73. Azmi H, Najwa H and Ennaji MM: Vitamin D Immunomodulatory Role in Chronic and Acute Viral Diseases. In: Emerging and Reemerging Viral Pathogens. Academic Press, pp489-506, 2020.

74. Beard JA, Bearden A and Striker R: Vitamin D and the anti-viral state. J Clin Virol 50: 194-200, 2011.

75. Greiller CL and Martineau AR: Modulation of the immune response to respiratory viruses by vitamin D. Nutrients 7: 4240-4270, 2015

76. Abhimanyu and Coussens AK: The role of UV radiation and vitamin D in the seasonality and outcomes of infectious disease. Photochem Photobiol Sci 16: 314-338, 2017.

77. Lang PO, Aspinall R and Vitamin D: Vitamin D status and the host resistance to infections: What it is currently (not) understood. Clin Ther 39: 930-945, 2017.

78. Gruber-Bzura BM: Vitamin D and influenza-prevention or therapy? Int J Mol Sci 19: 2419, 2018.

79. Rondanelli M, Miccono A, Lamburghini S, Avanzato I, Riva A, Allegrini P, Faliva MA, Peroni G, Nichetti M and Perna S: Self-Care for Common Colds: The pivotal role of Vitamin D, Vitamin C, Zinc, and Echinacea in three main immune interactive clusters (physical barriers, innate and adaptive immunity) involved during an episode of common colds-practical advice on dosages and on the time to take these nutrients/botanicals in order to prevent or treat common colds. Evid Based Complement Alternat Med 2018: 5813095, 2018.

80. Gombart AF, Pierre A and Maggini S: A review of micronutrients and the immune system-working in harmony to reduce the risk of infection. Nutrients 12: 236, 2020.

81. Teymoori-Rad M, Shokri F, Salimi V and Marashi SM: The interplay between vitamin D and viral infections. Rev Med Virol 29: e2032, 2019.

82. Shi YY, Liu TJ, Fu JH, Xu W, Wu LL, Hou AN and Xue XD: Vitamin D/VDR signaling attenuates lipopolysaccharide-induced acute lung injury by maintaining the integrity of the pulmonary epithelial barrier. Mol Med Rep 13: 1186-1194, 2016.

83. White JH: Vitamin D as an inducer of cathelicidin antimicrobial peptide expression: Past, present and future. J Steroid Biochem Mol Biol 121: 234-238, 2010.

84. Cantorna MT, Snyder L, Lin YD and Yang L: Vitamin D and 1,25(OH $)_{2} \mathrm{D}$ regulation of T cells. Nutrients 7: 3011-3021, 2015.

85. Wu D, Lewis ED, Pae M and Meydani SN: Nutritional modulation of immune function: analysis of evidence, mechanisms, and clinical relevance. Front Immunol 9: 3160, 2019.

86. Xu S, Chen YH, Tan ZX, Xie DD, Zhang C, Xia MZ, Wang H, Zhao H, Xu DX and Yu DX: Vitamin D3 pretreatment alleviates renal oxidative stress in lipopolysaccharide-induced acute kidney injury. J Steroid Biochem Mol Biol 152: 133-141, 2015.

87. Shi Y, Liu T, Yao L, Xing Y, Zhao X, Fu J and Xue X: Chronic vitamin $D$ deficiency induces lung fibrosis through activation of the renin-angiotensin system. Sci Rep 7: 3312, 2017.

88. Kong J, Zhu X, Shi Y, Liu T, Chen Y, Bhan I, Zhao Q, Thadhani R and Li YC: VDR attenuates acute lung injury by blocking Ang-2-Tie-2 pathway and renin-angiotensin system. Mol Endocrinol 27: 2116-2125, 2013.

89. Ferder M, Inserra F, Manucha W and Ferder L: The world pandemic of vitamin D deficiency could possibly be explained by cellular inflammatory response activity induced by the renin-angiotensin system. Am J Physiol Cell Physiol 304: C1027-C1039, 2013 
90. Ajabshir S, Asif A and Nayer A: The effects of vitamin D on the renin-angiotensin system. J Nephropathol 3: 41-43, 2014.

91. Li YC: Vitamin D regulation of the renin-angiotensin system. J Cell Biochem 88: 327-331, 2003.

92. Li YC, Kong J, Wei M, Chen ZF, Liu SQ and Cao LP: 1,25 -Dihydroxyvitamin $\mathrm{D}(3)$ is a negative endocrine regulator of the renin-angiotensin system. J Clin Invest 110: 229-238, 2002

93. Imai Y, Kuba K, Rao S, Huan Y, Guo F, Guan B, Yang P, Sarao R, Wada T, Leong-Poi H, et al: Angiotensin-converting enzyme 2 protects from severe acute lung failure. Nature 436: 112-116, 2005.

94. Treml B, Neu N, Kleinsasser A, Gritsch C, Finsterwalder T, Geiger R, Schuster M, Janzek E, Loibner H, Penninger J, et al: Recombinant angiotensin-converting enzyme 2 improves pulmonary blood flow and oxygenation in lipopolysaccharide-induced lung injury in piglets. Crit Care Med 38: 596-601, 2010

95. Nair R, Maseeh A and Vitamin D: Vitamin D: The 'sunshine' vitamin. J Pharmacol Pharmacother 3: 118-126, 2012.

96. Holick MF: The vitamin D deficiency pandemic: Approaches for diagnosis, treatment and prevention. Rev Endocr Metab Disord 18: 153-165, 2017.

97. Melamed ML, Michos ED, Post W and Astor B: 25-hydroxyvitamin D levels and the risk of mortality in the general population. Arch Intern Med 168: 1629-1637, 2008.
98. Mitchell F: Vitamin-D and COVID-19: Do deficient risk a poorer outcome? Lancet Diabetes Endocrinol 8: 570, 2020.

99. House N, Holborn H and Wc L: ICNARC report on COVID-19 in critical care. ICNARC 17: 1-26, 2020.

100. Ilie PC, Stefanescu S and Smith L: The role of vitamin D in the prevention of coronavirus disease 2019 infection and mortality. Aging Clin Exp Res 32: 1195-1198, 2020.

101. Mok CK, Ng YL, Ahidjo BA, Lee RC, Loe MW, Liu J, Tan KS, Kaur P, Chng WJ, Wong JE, et al: Calcitriol, the active form of vitamin $\mathrm{D}$, is a promising candidate for COVID-19 prophylaxis. bioRxiv: doi: https://doi.org/10.1101/2020.06.21.162396.

102. Daneshkhah A, Agrawal V, Eshein A, Subramanian H, Roy HK and Backman V: Evidence for possible association of vitamin D status with cytokine storm and unregulated inflammation in COVID-19 patients. Aging Clin Exp Res 32: 2141-2158, 2020.

(i) (9) This work is licensed under a Creative Commons Attribution-NonCommercial-NoDerivatives 4.0 International (CC BY-NC-ND 4.0) License. 